\title{
Key Role of Oxygen-Vacancy Electromigration in the Memristive Response of Ferroelectric Devices
}

\author{
C. Ferreyra, ${ }^{1,2}$ M. Rengifo, ${ }^{1,2}$ M.J. Sánchez $\odot,{ }^{1,3}$ A.S. Everhardt, ${ }^{4}$ B. Noheda $\odot,{ }^{4}$ and D. Rubi® ${ }^{1,2, *}$ \\ ${ }^{1}$ Instituto de Nanociencia y Nanotecnología (INN), CONICET-CNEA, Argentina \\ ${ }^{2}$ Centro Atómico Constituyentes, Av. Gral Paz 1499 (1650), San Martín, Buenos Aires, Argentina \\ ${ }^{3}$ Centro Atómico Bariloche and Instituto Balseiro (Universidad Nacional de Cuyo), 8400 San Carlos de \\ Bariloche, Ro Negro, Argentina \\ ${ }^{4}$ Zernike Institute for Advanced Materials, University of Groningen, Groningen 9747 AG, Netherlands
}

(Received 22 June 2020; revised 25 August 2020; accepted 21 September 2020; published 23 October 2020)

\begin{abstract}
Ferroelectric memristors are intensively studied due to their potential implementation in data storage and processing devices. In this work we show that the memristive behavior of metal-ferroelectricoxide-metal devices relies on the competition of two effects: the modulation of metal-ferroelectric interface barriers by the switchable ferroelectric polarization and the electromigration of oxygen vacancies, with the depolarizing field playing a fundamental role in the latter. We simulate our experimental results with a phenomenological model that includes both effects and we reproduce several nontrivial features of the electrical response, including resistance relaxations observed after external poling. Besides providing insight into the underlying physics of these complex devices, our work suggests that it is possible to combine nonvolatile and volatile resistive changes in single ferroelectric memristors, an issue that could be useful for the development of neuromorphic devices.
\end{abstract}

DOI: 10.1103/PhysRevApplied.14.044045

\section{INTRODUCTION}

Memristors are defined as capacitorlike structures displaying reversible and nonvolatile electrical resistance changes upon the application of electrical stimulus [1,2]. Besides their obvious potential for nonvolatile memories, memristors can mimic the behavior of brain synapses and are therefore expected to constitute one of the building blocks of neuromorphic computing devices [3,4] aiming to significantly enhance current electronic devices' performance in complex tasks such as pattern recognition or big data analysis. Memristive effects usually rely on the electromigration of defects such as oxygen vacancies $(\mathrm{O}-V)$, which can form conducting nanofilaments or modulate the height of Schottky barriers present at semiconductor-metal interfaces [1].

Ferroelectric memristors [5], where the resistance change is linked to the switching of the ferroelectric polarization direction, present a high interest due to the electronic nature of this effect, which is significantly faster than ionic migration mechanisms. Therefore, faster processing capabilities are expected for these kinds of devices. Ferroelectric memristive effects can be related to two mechanisms. On the one hand, for ultrathin ferroelectric layers (i.e., a few nanometers) sandwiched between

\footnotetext{
*diego.rubi@gmail.com
}

asymmetric metallic electrodes, usually called ferroelectric tunnel junctions (FTJs), electronic tunneling effects occur and the barrier height depends on the orientation of the ferroelectric polarization [5-8]. The barrier height change $\Delta \Phi$ is given by $\Delta \Phi=P\left(\lambda_{1}-\lambda_{2}\right) q / \epsilon$, where $P$ is the polarization, $\lambda_{1}$ and $\lambda_{2}$ are the electronic screening lengths for each electrode, $q$ is the electronic charge and $\epsilon$ is the permittivity of the ferroelectric layer. The change in the barrier height when the polarization is inversed produces a modification in the transmitted current for a given applied voltage, and in this way the resistance of the junction changes between two nonvolatile states. We also notice that FTJs require a by-design asymmetry (i.e., different metallic electrodes) to give different resistive states for both polarization orientations. Interestingly, it was recently proposed that a FTJ-like behavior was found in a oxide heterostructure with no ferroelectric layers, where a redox reaction between $\mathrm{MoSiO}_{x}$ and $\mathrm{YBCO}_{1-x}$ layers was highlighted as the dominant memristive mechanism for this system [9]. These results indicate that memristive effects related to oxygen electromigration-possibly entangled with the electronic tunneling effect - should not be ruled out in the study of oxide-based FTJs.

On the other hand, for devices involving thicker ferroelectric layers (i.e., tenths of nanometers) tunneling electronic transmission is negligible and the polarizationdependent memristive effect is usually attributed to the 
modulation of the Schottky barriers at ferroelectric-metal interfaces $[10-12]$. A polarization pointing to the Schottky interface leads into a decrease of the barrier height, resulting in a low-resistance state. When the polarization is inversed, the barrier height increases and a high-resistance state is stabilized. The dependence of interface energy barriers with the ferroelectric polarization has been inferred from spectroscopic techniques [13,14], transport measurements $[15,16]$, and has also been theoretically simulated [17]. Other relevant parameters of ferroelectric-metal contacts such as built-in potential, charge density, and depletion width have also been theoretically modeled [18]. A memristive effect could also be present in symmetric metal-ferroelectric-metal devices where two complementary interfaces can display memristive behavior simultaneously, as was previously shown for ionic migration-based memristive systems [19].

Different reports on ferroelectric memristors evidence a relaxation of the ideally nonvolatile resistive states with time, with a typical time scale of minutes [20,21]. This constitutes a severe drawback to the implementation of ferroelectric memristors in devices where retention times are a key issue.

One possible scenario to explain the resistance relaxations in ferroelectic memristors is related to a loss of polarization with time due to the existence of a strong depolarizing field [22]. On the other hand, it was shown that ferroelectric memristive effects coexist with $\mathrm{O}-V$ migration in different systems [23-27]. For instance, Qian et al. reported that for $\mathrm{BaTiO}_{3}$-based ferroelectric memristors, two different mechanisms contribute to electroresistance (ER) [25]. The knob that controls the appearance of one or the other mechanism was the time length of the writing pulses; for short pulses $(20 \mu \mathrm{s})$ the memristive behavior was dominated by the modulation of the Schottky barrier by the direction of the ferroelectric polarization (that is, an electronic effect), but for longer pulses (20 s) $\mathrm{O}-\mathrm{V}$ electromigration seemed to dominate the change of the Schottky barrier height over the mentioned electronics effects. Both regimes were clearly differentiated from remanent resistance versus voltage curves with different circulations and distinct temperature behavior. For writing pulses with intermediate time lengths it is reasonable to expect the coexistence of both effects. Very recently, it was proposed that a polarization-coupled charge trapping and detrapping mechanism also participates in the memristive response of $\mathrm{BiFeO}_{3}$ devices [28].

These results indicate that further efforts should be performed to disentangle coexisting mechanisms related to the electrical response of ferroelectric memristors. With this aim, in the present work we show, in the case of symmetric memristive metal-ferroelectric oxide-metal systems, that distinct features of the remanent resistance versus voltage loops can be rationalized in terms of a competition between electronic effects, linked to the polarization inversion, and the electromigration of $\mathrm{O}-\mathrm{V}$, both modulating the metal-ferroelectric Schottky barriers. A similar electrical response was found for two devices with different ferroelectric oxides $\left(\mathrm{Pb}\left(\mathrm{Zr}_{0.20} \mathrm{Ti}_{0.80}\right) \mathrm{O}_{3}\right.$ and $\left.\mathrm{BaTiO}_{3}\right)$, electrodes ( $\mathrm{Pt}$ and $\mathrm{SrRuO}_{3}$, respectively) and geometries (out-of-plane and in-plane configurations, respectively), suggesting that the scenario presented here could be a general one for ferroelectric memristors with interfacial resistive switching [10-12].

Aided by phenomenological simulations that reproduce several nontrivial features of the experimental memristive response, we show that the driving force for O- $V$ electromigration in the absence of external electrical stimulus is the depolarizing field present on the ferroelectric due to incomplete charge screening by the metallic electrodes. Moreover, our work provides strong evidence and physical insight about the coexistence of both nonvolatile and volatile resistive changes in ferroelectric based devices, an issue that might be useful for the development of neuromorphic computing devices.

\section{EXPERIMENTAL DETAILS}

We characterize two metal-ferroelectric-metal systems: a commercial $\mathrm{Pt} / \mathrm{Pb}\left(\mathrm{Zr}_{0.20} \mathrm{Ti}_{0.80}\right) \mathrm{O}_{3} / \mathrm{Pt}$ capacitor (Pt/PZT/Pt, Radiant Technologies AB ferroelectric capacitor) and a $\mathrm{SrRuO}_{3} / \mathrm{BaTiO}_{3} / \mathrm{SrRuO}_{3}(\mathrm{SRO} / \mathrm{BTO} / \mathrm{SRO})$ device. In the first case, the polycrystalline PZT layer is $255 \mathrm{~nm}$ thick, the Pt bottom and top electrodes are 150 and $100 \mathrm{~nm}$ thick, respectively, and the device area is $10^{5} \mu \mathrm{m}^{2}$. Polarization-voltage loops, typical of a standard ferroelectric device, can be found in Ref. [29]. In the second case, epitaxial BTO thin films (90 nm thick) are deposited by pulsed-laser deposition on $\mathrm{NdScO}_{3}$ substrates. After that, interdigital SRO/Pt top electrodes are deposited and shaped by standard lithographic techniques. The device geometry is shown in Fig. 1(b). Electrical measurements are performed along the BTO (100) in-plane direction. The planar geometry of the BTO device is chosen due to its standard ferroelectric behavior (see the corresponding polarization-voltage loops recorded on the same samples in Ref. [30]), which eases the understanding of the memristive response. This also allows a direct comparison with the Pt/PZT/Pt device. The ferroelectric properties of out-of-plane $\mathrm{NdScO}_{3} / \mathrm{SRO} / \mathrm{BTO} / \mathrm{SRO}$ devices display an atypical behavior [30], characterized by a $P-V$ loop with no remanence and $I-V$ curves with multiple switching currents. The study of the memristive behavior of out-of-plane devices escapes the scope of the present paper. Further exhaustive characterization of the BTO-based structures reported here can be found in Refs. [30-32].

Given the large values of the two-point resistance of the in-plane BTO devices (approximately equals $100 \mathrm{G} \Omega$ ), which requires measuring dc currents (approximately equals $10 \mathrm{fA}$ ) below the sensitivity of our source 
(a)

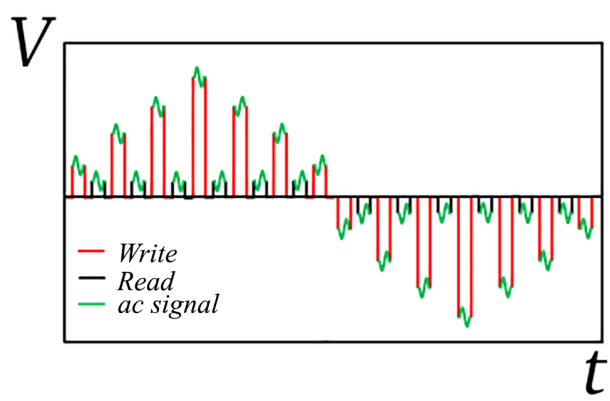

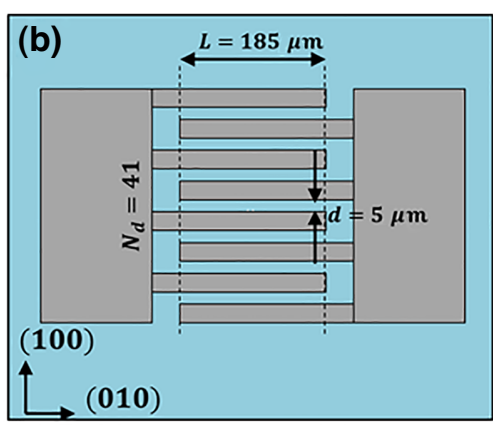

(d) (c)

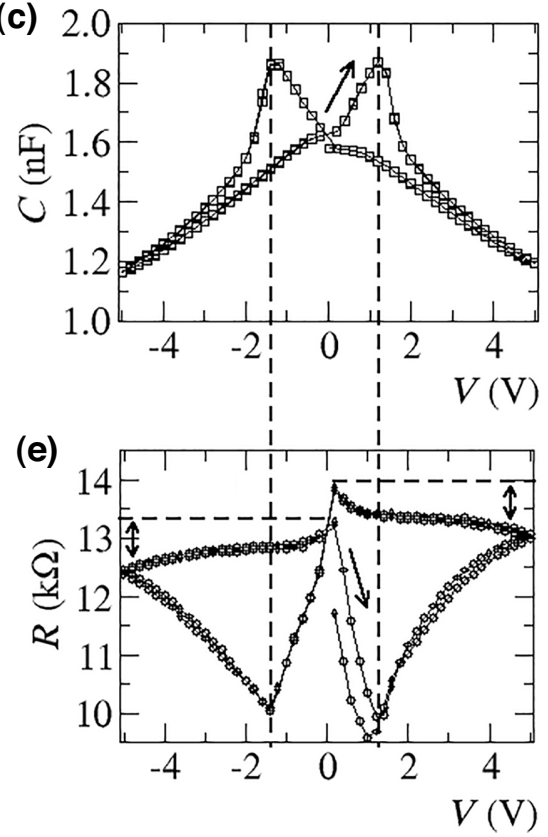

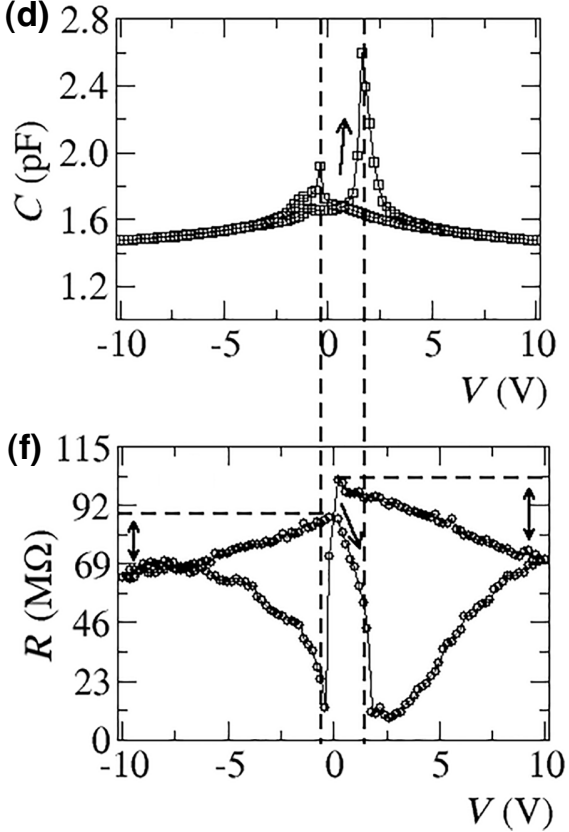

FIG. 1. (a) Sketch showing the stimuli protocol used to measure simultaneously $C-V$ curves and HSLs in our samples. (b) Sketch displaying a top view of the interdigital Pt/SRO electrodes on top of BTO thin films. $N_{d}$ corresponds to the number of fingers of the electrodes. $C-V$ curves measured on $\mathrm{Pt} / \mathrm{PZT} / \mathrm{Pt}$ (c) and SRO/BTO/SRO (d) devices. HSLs recorded on Pt/PZT/Pt (e) and SRO/BTO/SRO (f) devices. See text for details. Single arrows indicate the evolution of the curves. meters, we perform electrical ac measurements, which allow a straightforward comparison between the electrical response of both PZT and BTO devices. We record, simultaneously, capacitance-voltage $(C-V)$ and remanent resistance-voltage (usually called hysteresis switching loops or HSLs) curves from ac measurements by using a standard $L C R$ meter set in $R C$ parallel circuit mode, which allows extracting values for $R$ and $C$.

The stimuli protocol consists on the application of alternated writing and (undisturbing) reading dc pulses, with a superimposed small ac signal in both cases (frequencies $f$ between $100 \mathrm{~Hz}$ and $1 \mathrm{MHz}$ are tested), as shown in Fig. 1(a). The time width of writing pulses is $\tau_{W} \approx 50 \mathrm{~ms}$ and the time between two consecutive writing pulses is $\Delta \tau \approx$ $4 \mathrm{~s}$. The $C$ - $V$ curve is extracted from the capacitances measured during the application of the writing pulses, while the HSL is extracted from the resistances measured during the application of reading pulses. The dc bias of the reading pulses is $200 \mathrm{mV}$ and the ac amplitude - typically a few hundred $\mathrm{mV}$ - is chosen at each frequency to maximize the signal-to-noise ratio of the HSL. We notice that the (ac) measurement of dynamic $C-V$ curves (that allows determining the coercive field $E_{C}$ ) and remanent HSL (necessary to track the memristive behavior) in the same run permits proving that both ferroelectric and resistance switching take place after the same applied writing voltage, providing evidence about the link between memristive behavior and ferroelectricity [33]. We recall that key figures of the ferroelectric response, such as $E_{C}$, might be sensitive to subtle details of the measurement protocol [34], so our procedure avoids difficulties in correlating capacitance and remanent resistance measurements taken with different equipment or protocols.

The $C-V$ curves $(f=100 \mathrm{kHz})$ for $\mathrm{Pt} / \mathrm{PZT} / \mathrm{Pt}$ and SRO/BTO/SRO devices are shown in Figs. 1(c) and 1(d), respectively, displaying in both cases the typical butterflylike shape of ferroelectric systems. A slight imprint is found in the case of the SRO/BTO/SRO device.

Figures 1(e) and 1(f) display HLSs recorded for $\mathrm{Pt} / \mathrm{PZT} / \mathrm{Pt}$ and SRO/BTO/SRO devices, respectively, also for $f=100 \mathrm{kHz}$. In both cases, the so-called "table with legs" is found [19], typical of two series complementary memristive interfaces; that is, when one interface switches from low resistance (LR) to high resistance (HR), the other 
switches inversely. Two series switchable Schottky interfaces (Pt/PZT and SRO/BTO, respectively [35-37]) are likely at the origin of the memristive behavior in each system. We notice that in both cases, the "legs" in Figs. 1(e) and 1(f) match well with the capacitance peaks related to ferroelectric switching observed, respectively, in Figs. 1(c) and 1(d). This suggests the existence of ferroelectric memristive behavior; that is, each interface's Schottky barrier is modulated by the direction of the ferroelectric polarization.

However, a distinct feature is observed in both HSLs: the resistance of the top part of the "tables" presents a noticeable positive (negative) slope for negative (positive) voltages. This behavior is at odds with the one exhibited by (nonferroelectric) symmetric memristive complementary interfaces $[19,38]$, where the "table with legs" HSLs exhibit a flat "table" (that is zero slope for both positive and negative voltages), as sketched by the horizontal dotted lines in Figs. 1(e) and 1(f). This quite unusual feature suggests that each interface resistance is modulated by the competition of ferroelectric memristive effects and the electromigration of $\mathrm{O}-V$. A similar interpretation was done by $\mathrm{Hu}$ et al. from the observation of a nonsquared HSL in $\mathrm{BiFeO}_{3}$-based FTJ [39]. Going back to our experiments, we recall that HSLs for the Pt/PZT/Pt system are recorded in a broad frequency range between $100 \mathrm{~Hz}$ and $1 \mathrm{MHz}$ and display similar features as those already described $[40,41]$.

In order to get further insight into the different scenarios and explain our experimental HLSs, we perform numerical simulations based on the voltage-enhanced O- $V$ drift (VEOV) model, developed originally to describe nonferroelectric perovskites $[19,42,43]$, further extended to simple oxide-based $[38,44]$ memristive devices and here adapted to a symmetric metal-ferroelectric-metal system, as shown below.

\section{SIMULATIONS}

To simulate the memristive behavior of the devices, we consider a symmetric metal-ferroelectric-metal $(M-F-M)$ system where both metal-ferroelectric interfaces are Schottky type and connected in a back-to-back configuration. We assume that the barrier heights depend on the direction of the electrical polarization. We recall that if the depolarizing field $E_{\mathrm{DP}}$ is close to or higher than the coercive field $E_{C}$, the ferroelectric could be spontaneously depolarized [45] or even ferroelectricity could vanish for ultrathin films [46], otherwise both the polarization $P$ and $E_{\mathrm{DP}}$ should be steady with time. The Pt/PZT/Pt device is shown to display polarization retention times higher than $10^{5} \mathrm{~s}$ with no significant polarization loss [29]. This time range is much larger than the typical time range of the electrical characterization experiments we report in this paper. On the other hand, the exhaustive characterization previously performed in our BTO devices [30-32] showed no sign of polarization loss with time. This indicates that for our two ferroelectric devices both $P$ and $E_{\mathrm{DP}}$ are stable with time between ferroelectric switching events, as we assume for the modeling.

We assume that both PZT and BTO devices contain $\mathrm{O}-\mathrm{V}$, which also modulate the height of Schottky barriers present at metal-ferroelectric interfaces. The presence of $\mathrm{O}-\mathrm{V}$ in the BTO structures studied here was previously determined by some of us from x-ray diffraction experiments in Ref. [31]. This indicates that the films present an $\mathrm{O}-V$ density of at least approximately equal to $5 \times 10^{20} \mathrm{~cm}^{-3}$ [47]. In the case of the PZT device it was shown that it is prone to fatigue [29], an effect that is usually ascribed to $\mathrm{O}-V$ redistribution upon electrical cycling $[48,49]$. In addition, we recall that memristive effects in PZT related to O- $V$ electromigration were previously reported [50]. The issues described above, plus the similarity of the memristive response with respect to BTO devices, provide reasonable evidence of the presence of $\mathrm{O}-V$ in the PZT device.

We assume that the presence of $\mathrm{O}-V$ locally reduces the resistivity of the ferroelectric oxide, as is the case of BTO [51], PZT [52], and other nonferroelectric simple oxides, such as $\mathrm{TiO}_{2}$ [44]. Bearing these considerations in mind, we propose the following phenomenological equation for the device two-point resistance:

$$
R^{T}=R^{\mathrm{eff}} A^{F}(P) M^{\mathrm{O}-V}
$$

where $R^{\text {eff }}$ is an effective (constant) resistance, while $A^{F}(P)$ and $M^{\mathrm{O}-V}$ are two adimensional factors. $A^{F}(P)$ modulates the interface resistances and is a function of the actual ferroelectric polarization $\vec{P}$, while $M^{\mathrm{O}-V}$ depends on the O- $V$ distribution along the device, as we show below.

We start by deriving the relation between $A^{F}$ and the device polarization $\vec{P}$ (which we assume as single domain) by considering, in the first place, a scenario with no $\mathrm{O}-\mathrm{V}$ electromigration. As customary in memristive devices, the HSL is constructed by applying a read pulse $V^{R}$ after the write one $V^{W}$, which induces the resistive change. $V^{R}$ is small enough to warrant that both Schottky interfaces, named $L$ and $R$, respectively, remain reversely biased. The current $I$ for a Schottky interface biased with a voltage $V$ can be written as [53]

$$
I=\beta(V, T) \exp \left[-\frac{e}{k_{B} T} \phi_{\alpha}\right]
$$

where $\beta(V, T)$ is a constant for given temperature $T$ and voltage bias $V$. The barrier height $\phi_{\alpha}$ for a metalferroelectric Schottky junction can be expressed as $[14,54]$

$$
\phi_{\alpha}=\phi_{\alpha}^{0} \mp \gamma|\vec{P}|,
$$

where $\phi_{\alpha}^{0}$ is the bare barrier height-related to the difference between the metal work function and the insulator 


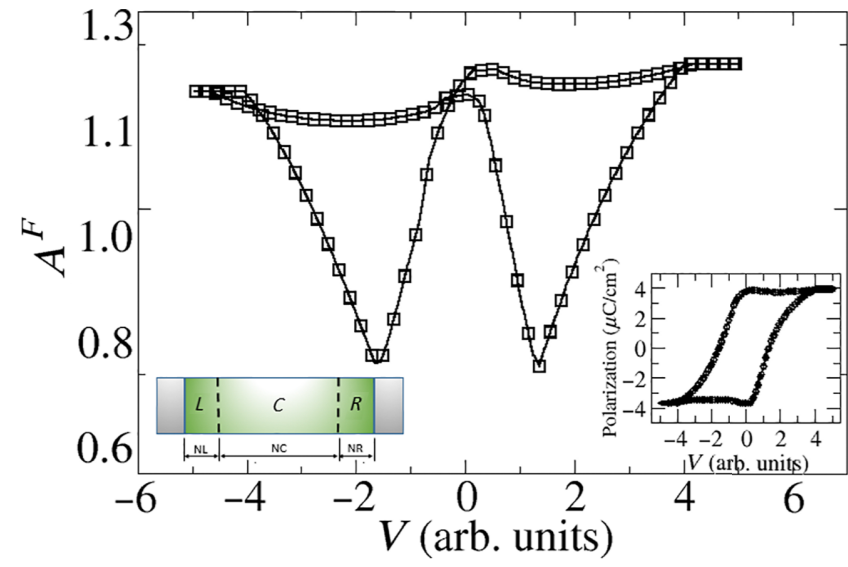

FIG. 2. $A^{F}$ vs $V^{W}$, where $A^{F}$ is defined in Eq. (6) (the value of $\tilde{\gamma}$ is given in Table I). Left inset: sketch of the metal-ferroelectricmetal device, where $L, C$, and $R$ zones defined for our simulations are explicitly shown. NL, NC, and NR correspond to the number of nanodomains present in each zone, respectively. Right inset: $P-V$ loop obtained after integration of the experimental $C-V$ data for the $\mathrm{Pt} / \mathrm{PZT} / \mathrm{Pt}$ system. Notice that the voltage scale of the $P-V$ loop is converted according to $1 \mathrm{~V}=1 \mathrm{arb}$. units in order to match the voltage scale of the simulation showed in the main panel.

electron affinity - and $\mp \gamma|\vec{P}|$ is the correction due to the presence of ferroelectric bound charges at the interface. If the polarization points to the Schottky interface then the barrier height decreases, while in the opposite case the barrier height increases.

We now consider the symmetric $M-F-M$ system comprising the two series back-to-back Schottky interfaces $L$ and $R$, as sketched in the inset of Fig. 2. We assume for the polarization $P$ a positive value when pointing from $L$ to $R$, and a negative value otherwise. Thus, when $P$ goes from $L$ to $R$, the barrier height at interface $L$ increases while at interface $R$ reduces. In this case, the current $I$ is limited by the barrier $L$, where most of the voltage drop due to $V^{R}$ occurs, and according to the previous convention for the polarization sign, it can be expressed as $I \sim I_{L}=\beta \exp \left[-e / k_{B} T\left(\phi_{\alpha}^{0}+\gamma_{L} P\right)\right]$.

On the other hand, when the polarization is reversed $(P<0)$, the current is limited by interface $R$ and thus given by $I \sim I_{R}=\beta \exp \left[-e / k_{B} T\left(\phi_{\alpha}^{0}-\gamma_{R} P\right)\right]$.

The last two equations can be written in compact form as

$$
I=\beta \exp \left[-\frac{e}{k_{B} T}\left(\phi_{\alpha}^{0}+\gamma|P|\right)\right]=I_{0} \exp (-\tilde{\gamma}|P|),
$$

where we assume that both interfaces are identical $\left(\gamma_{L}=\gamma_{R}=\gamma\right)$ and we define the constants $I_{0}=$ $\beta \exp \left[-e / k_{B} T\left(\phi_{\alpha}^{0}\right)\right]$ and $\tilde{\gamma}=\left(e / k_{B} T\right) \gamma$, respectively.

Equation (4) explicitly shows the dependence of the current $I$ along the device with the polarization $P$.
Under the assumption that the device resistance is dominated by the interfaces and solely modulated by the direction of the ferroelectric polarization, a remanent two-point resistance $R(P)$ can be computed as

$$
R(P) \approx \frac{V^{R}}{I_{0} \exp (-\tilde{\gamma}|P|)}=\frac{R_{0}}{\exp (-\tilde{\gamma}|P|)},
$$

where $R_{0}=V^{R} / I_{0}$, with resistance dimension. By comparing Eqs. (1) and (5), and for the scenario with no O- $V$ electromigration considered so far, we assume $R^{T} \approx R(P)$ and thus $R_{0}=R^{\mathrm{eff}} M_{0}^{\mathrm{O}-V}$, where $M_{0}^{\mathrm{O}-V}$ is a constant determined by the initial O- $V$ configuration. Therefore, we obtain

$$
A^{F}(P) \equiv \frac{1}{\exp (-\tilde{\gamma}|P|)},
$$

which gives the modulation of the device resistance with the amplitude and direction of the ferroelectric polarization $\vec{P}$.

To compute the evolution of $A^{F}$ with $P$ upon voltage cycling, it is necessary to know the dependence of $P$ on $V^{W}$. To this end, we use the $P-V$ loop corresponding to the $\mathrm{Pt} / \mathrm{PZT} / \mathrm{Pt}$ device, obtained after integrating the experimental $C-V$ data [Fig. 1(c)], which shows a more symmetric behavior than the $C$ - $V$ curve corresponding to the SRO/BTO/SRO device [Fig. 1(d)].

The $P-V$ loop is shown in the (right) inset of Fig. 2, while in the main panel the calculated $A^{F}$ vs $V^{W}$ is displayed. The latter figure can be interpreted (up to dimensional prefactors) as a HSL obtained under the assumption that the memristive effect relies exclusively on the modulation of both Schottky barriers by the switchable ferroelectric polarization. The curve is qualitatively similar to standard HSLs reported for symmetric memristive systems $[19,38,55]$ in which the resistance switching is based on a completely different mechanism involving $\mathrm{O}-\mathrm{V}$ electromigation.

In our case, the obtained $A^{F}$ vs $V^{W}$ is a demonstration of the complementary behavior in $M-F$ interfaces. However, it significantly differs from the experimental HSL displayed in Fig. 1(e), mainly in the slopes of the top zones of the "table," as already described in Sec. II. This indicates the need of including an additional ingredient to properly simulate the behavior of our device.

In the following, we show that by taking into account the electromigration of $\mathrm{O}-V$ in the modeling as a competitive memristive effect with the ferroelectric one, allows us to properly collect the physics of our devices.

To determine the $M^{\mathrm{O}-V}$ factor in Eq. (1), related to O- $V$ electromigration, we consider a unidimensional path across the ferroelectric, which bridges both electrodes and is divided in the three zones: the central one $C$, composed of NC nanodomains, and the left $(L)$ and right 
$(R)$ zones, having NL and NR nanodomains, respectively, comprising the interfaces with the metallic electrodes. The total number of nanodomains is thus given by $N=\mathrm{NL}+$ $\mathrm{NC}+\mathrm{NR}$.

To each nanodomain we assign a site $i$ and define its resistivity, $\rho_{i}$, in terms of the local density of O-V, $\delta_{i}$. According to Refs. $[38,44]$ we write

$$
\rho_{i}^{\mathrm{O}-V}=\rho_{0}\left(1-A_{i} \delta_{i}\right),
$$

where $\rho_{0}$ is the resistivity in the absence of O- $V$. The factors $A_{i}$ satisfy $A_{i} \delta_{i}<1 \forall i$, and for simplicity are taken as constants along each specific region $L, C$, and $R$, respectively. In the simulations we consider $A_{L} \sim A_{R}<A_{C}$, to capture the fact that the regions close to the Schottky barriers are more resistive that the central $C$ zone. We assume that the device presents initially an amount of $\mathrm{O}-V$ giving a total density of $\delta_{0}=0.16$ (total number of O- $V$ divided by the number of sites $N$ ), that remains constant during subsequent electrical cycling. This is a reasonable assumption as the $\mathrm{Pt} / \mathrm{PZT} / \mathrm{Pt}$ device is encapsulated and, for the case of the SRO/BTO/SRO device, SRO has been shown to act as a blocking barrier to oxygen diffusion [56]. This implies that oxygen exchange with the environment is negligible in both cases, and $\mathrm{O}-V$ migration is only possible within the device.

For a given $\mathrm{O}-V$ configuration, the two-point resistance $R^{\mathrm{O}-V}$ of the complete domains' path is

$R^{\mathrm{O}-V}=R_{0}^{\prime}\left(N-\sum_{i=1}^{\mathrm{NL}} A_{L} \delta_{i}-\sum_{i=\mathrm{NL}+1}^{\mathrm{NL}+\mathrm{NC}} A_{C} \delta_{i}-\sum_{i=N-\mathrm{NR}+1}^{N} A_{R} \delta_{i}\right)$,

where we define the resistance $R_{0}^{\prime}=\rho_{0} \mathcal{L}$, where $\mathcal{L}$ is the typical length of each nanodomain.

We now define the adimensional prefactor $M^{\mathrm{O}-V}$ appearing in Eq. (1) as

$$
M^{\mathrm{O}-V} \equiv \frac{R^{\mathrm{O}-V}}{R_{0}^{\prime}},
$$

which is computed straightforwardly from Eq. (8), once $R^{\mathrm{O}-V}$ is known.

In the VEOV model [19], the probability rate $p_{i j}$ for an $\mathrm{O}-V$ to hop from one site to a neighbor site is given by

$$
p_{i j}=\delta_{i}\left(1-\delta_{j}\right) \exp \left(-V_{0}+\Delta V_{i}\right)
$$

where $\delta_{i}$ and $\delta_{j}$ are the O- $V$ densities at sites $i$ and $j$, respectively. We define $V_{0}$ as the activation energy for $\mathrm{O}-V$ diffusion - we take as $V_{0 L}, V_{0 C}$, or $V_{0 R}$ depending on the region $L, C$, or $R$, respectively - and $\Delta V_{i}$ as the voltage drop at site $i$. Both are adimensional quantities written in terms of the thermal energy $k_{B} T$.
In the following we assume that $\Delta V_{i}$ can be written as the sum of two terms. The first one is the voltage drop due to the external bias $V^{W}$ and the second is the contribution from a depolarizing voltage arising from the existence of a depolarizing field $E_{\mathrm{DP}}$ due to incomplete screening of the ferroelectric bound charges at both $M-F$ interfaces [57].

We assume for the depolarizing field $E_{\mathrm{DP}}=-\beta P$ [58], where $P$ is the ferroelectric polarization and $\beta$ a constant.

When a voltage is applied to the device, the behavior of screening charges is rather complex as a consequence of the interplay between polarization, screening, and injected charges (see Ref. [59] and references therein). However, it is usually a good approximation to consider that the total field acting in the volume of the biased ferroelectric is the superposition of the external field and $E_{\mathrm{DP}}$ in shortcut conditions. This approximation was made, for example, in Ref. [45] to experimentally extract $E_{\mathrm{DP}}$ in BTO-biased devices from polarization-relaxation experiments and is the approach we use in our modeling. Therefore, the voltage drop $\Delta V_{i}$ at site $i$ is written as

$$
\Delta V_{i}=V^{W} \frac{R_{i}^{\mathrm{O}-V}}{R^{\mathrm{O}-V}}+E_{\mathrm{DP}} \mathcal{L}=V^{W} \frac{R_{i}^{\mathrm{O}-V}}{R^{\mathrm{O}-V}}-\xi P,
$$

where $R_{i}^{\mathrm{O}-V}=\rho_{i}^{\mathrm{O}-V} \mathcal{L}$ and $\xi=\beta \mathcal{L}$.

Given an external voltage $V^{W}(t)$ applied at time $t$, the O- $V$ density at site $i$ is updated for each simulation step according to Eqs. (10) and (11).

In order to restrict the dynamics of $\mathrm{O}-V$ to the active region, we take the rates $p_{01}=p_{10}=p_{N N+1}=p_{N+1 N}=0$. In addition, as the total number of $\mathrm{O}-V$ is conserved, it is satisfied that $\sum_{i=1}^{N} \delta_{i}=\delta_{0}, \forall t$.

To match the experiments, we choose the stimulus $V^{W}(t)$ as a linear ramp following the cycle $0 \rightarrow V_{m 1}^{W} \rightarrow-V_{m 2}^{W}$ $\rightarrow 0$. At each simulation time step $t_{k}$ we compute the local voltage drops $\Delta V_{i}\left(t_{k}\right)$ and employing the probability rates $p_{i j}$ we obtain the transfers between nearest neighboring sites. Afterwards the values $\delta_{i}\left(t_{k}\right)$ are updated to a new set of densities $\delta_{i}\left(t_{k+1}\right)$, with which we compute, at time $t_{k+1}$, the local resistivities $\rho_{i}\left(t_{k+1}\right)$, the local voltage drops under the applied voltage $V^{W}\left(t_{k+1}\right)$ and the factor $M^{\mathrm{O}-V}\left(t_{k+1}\right)$ from Eq. (9), to start the next simulation step at $t_{k+1}$. For the polarization $P\left[V^{W}\left(t_{k}\right)\right] \equiv P\left(t_{k}\right)$ we use the $\mathrm{Pt} / \mathrm{PZT} / \mathrm{Pt}$ experimental curve given by Fig. 2.

Following this algorithm, we compute the evolution of the $M^{\mathrm{O}-V}$ vs $V^{W}$, which accounts in Eq. (1) for the memristive effect related to O- $V$ electromigration due to the action of both the external voltage and the internal depolarizing field.

We recall that in standard memristive systems driven by $\mathrm{O}-V$ electromigration the time widths and intensity of writing pulses are key parameters that tune the memristive response [60]. For the present ferroelectric system, the dynamics of $\mathrm{O}-V$ are dependant on two competitive effects. When the write voltage $V^{W}$ is on, O- $V$ drift in one direction 
(i.e., parallel to the applied external electrical field, which also aligns the polarization in the same direction). When $V^{W}$ is turned off, O- $V$ electromigration is solely ruled by the depolarizing field, and thus in the direction antiparallel to the polarization.

The time windows in which the external voltage is switched on and off, respectively, are therefore critical for the overall O- $V$ dynamics. To collect these features in our simulations, we simulate three situations with $\tau_{W} / \tau_{\text {off }}=$ $1 / 99,1 / 9$, and $1 / 4$, where $\tau_{W}$ and $\tau_{\text {off }}$ are the on and off external voltage time windows, respectively. We assume in all cases that two consecutive write pulses are separated by a time width of $1000 \mathrm{arb}$. units.

The numerical values of the parameters used in the simulation are shown in Table I. These values correspond to the $\mathrm{Pt} / \mathrm{PZT} / \mathrm{Pt}$ device and are mostly assigned based on physical considerations. For example, O- $V$ activation energies $V_{0 i}$ are $0.06-0.08 \mathrm{eV}$, which are close to the bottom limit of the range of values reported for perovskites (0.2-1.2 eV [61], 0.1-0.15 eV [62]). The assumed activation energies are closer to the single ionized vacancy values reported for PZT, with double ionized vacancies presenting much larger energies, up to $1 \mathrm{eV}$ [63]; the value used for $\xi=2500 \mathrm{~J} \mathrm{~cm}^{2} / \mathrm{C}^{2}$ leads to a depolarizing field $E_{\mathrm{DP}} \approx 3.9 \mathrm{MV} / \mathrm{m}$ (see Eq. 11) and allows extracting, assuming a Thomas-Fermi screening [64], a screening length of $\mathrm{Pt} \approx 0.1 \mathrm{~nm}$, close to the typical values for metals; the number of $\mathrm{O}-\mathrm{V}$ used in the model is consistent with a density $C_{\mathrm{OV}} \approx 3 \times 10^{17} \mathrm{~cm}^{-3}$, which is within the range reported for titanates [47]. The values of $A_{i}$ are assumed $A_{L, R}<A_{C}$ to reflect the more resistive nature of

TABLE I. Table with the numeric values of the different parameters used in the simulations. The equations where the parameters are used are numbered as in the main text and shown in the right column.

\begin{tabular}{lcc}
\hline \hline Parameters & Value & Equation \\
\hline $\mathcal{L}$ & $2.55 \mathrm{~nm}$ & $(11)$ \\
$\mathrm{NL}$ & $61.20 \mathrm{~nm}$ & $(8)$ \\
$\mathrm{NC}$ & $132.60 \mathrm{~nm}$ & $(8)$ \\
$\mathrm{NR}$ & $61.20 \mathrm{~nm}$ & $(8)$ \\
$\tilde{\gamma}$ & $1.06 .10^{5} \mathrm{~cm}^{2} / \mathrm{C}$ & $(4)$ \\
$R^{\mathrm{eff}}$ & $160 \Omega$ & $(1)$ \\
$R_{0}^{\prime}$ & $129 \Omega$ & $(8)$ \\
$R_{0}$ & $11.60 \mathrm{k} \Omega$ & $(5)$ \\
$A_{L}$ & 10.40 & $(8)$ \\
$A_{C}$ & 26.40 & $(8)$ \\
$A_{R}$ & 11.04 & $(8)$ \\
$V_{0 L}$ & $(10)$ \\
$V_{0 C}$ & $2.9^{\mathrm{a}}(0.07 \mathrm{eV})$ & $(10)$ \\
$V_{0 R}$ & $2.3^{\mathrm{a}}(0.06 \mathrm{eV})$ & $(10)$ \\
$\xi$ & $3.2^{\mathrm{a}}(0.08 \mathrm{eV})$ & $(11)$ \\
\hline \hline
\end{tabular}

a The parameters $V_{0 L}, V_{0 C}$, and $V_{0 R}$ are normalized in units $k_{B} T=$ $0.025 \mathrm{eV}$ (room temperature). metal-ferroelectric interfaces due to the presence of energy barriers [44] and disorder [38]. The only free parameter that is extracted from a quantitative fitting is $\mathrm{R}^{\mathrm{eff}}$, as shown later.

In Fig. 3(a) we show the evolution of $M^{\mathrm{O}-V}$ as a function of $V^{W}$, without including the effect of the depolarizing field (i.e., perfect screening at the electrodes) for these three situations. It is found that for $\tau_{W} / \tau_{\text {off }}=1 / 4$ there is a large hysteresis in the curve, for $\tau_{W} / \tau_{\text {off }}=1 / 9$ the hysteresis is mild while no hysteresis is found for $\tau_{W} / \tau_{\text {off }}=1 / 99$. This indicates that, as expected, longer external stimulus induces larger $\mathrm{O}-V$ electromigration in the direction parallel to the external stimulus, similar to the case of nonferroelectric systems.

Figure 3(b) analyzes a similar situation now including the presence of the depolarizing field $E_{\mathrm{DP}}$ (again, the parameters are shown in Table I). In this case, unlike the previous one, there is an evident large hysteresis for the
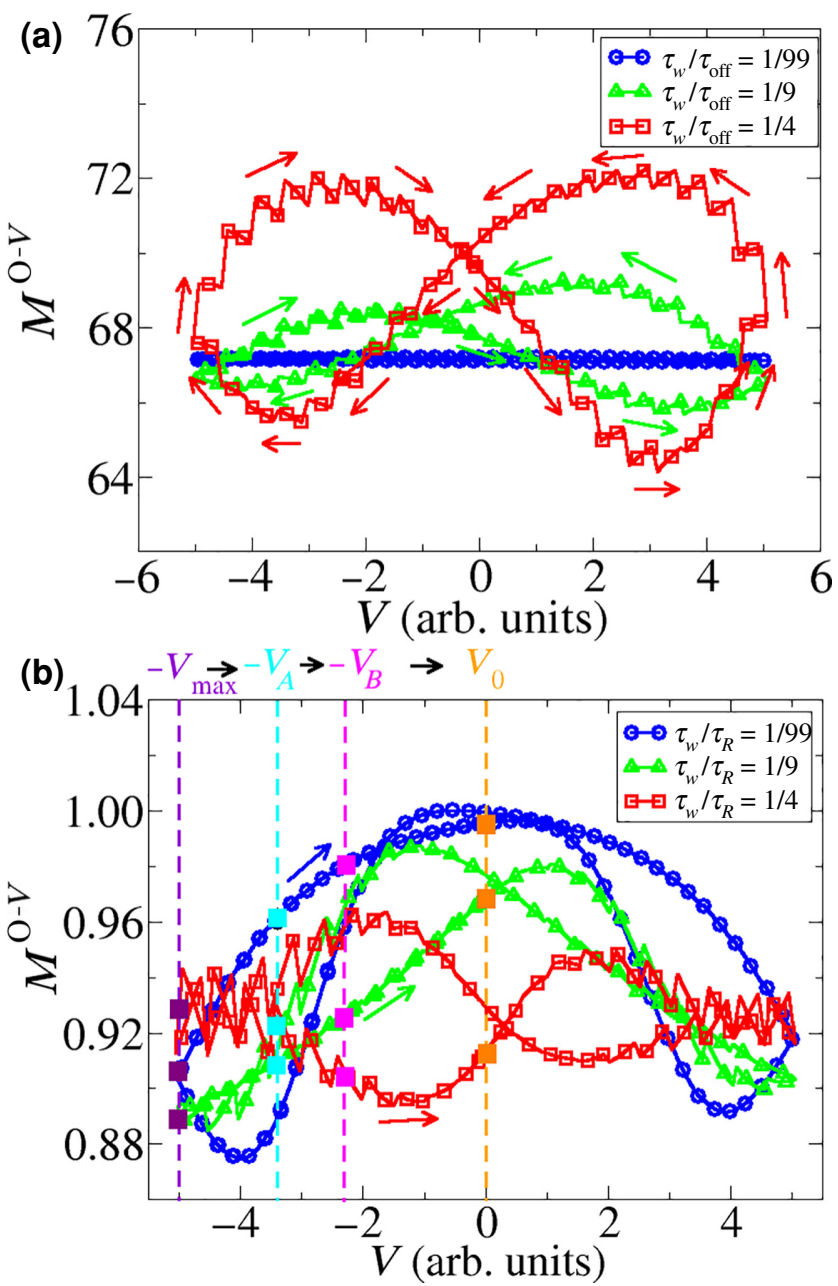

FIG. 3. Evolution of $M^{\mathrm{O}-V}$ vs $V^{W}$ for different $\tau_{W} / \tau_{\text {off }}$ ratios, shown in the respective legends. (a) Under the sole action of the external stimuli (voltage). (b) Including the effects of both the external stimulus and the depolarizing field. 
three situations. We notice that for $\tau_{W} / \tau_{\text {off }}=1 / 99$ and $1 / 9$ the curve circulations are inverted with respect to the one corresponding to $1 / 4$. This crossover is an indication of a change in the O- $V$ dynamics between shorter and longer pulses, where the effect of the depolarizing field should become more pronounced as $\tau_{W}$ is reduced. In order to shed light on this issue, Figs. 4(a)-4(c) display the O- $V$ profiles for the explored $\tau_{W} / \tau_{\text {off }}$ ratios, after the application of writing pulses $-V_{\max } \rightarrow-V_{A} \rightarrow-V_{B} \rightarrow V_{0}=0$, as indicated in Fig. 3(b). Notice that the color code of the O- $V$ profiles of Figs. 4(a) -4(c) corresponds to the $V_{i}$ colors in Fig. 3(b).

The analysis of Figs. 4(a)-4(c) shows that for all $\tau_{W} / \tau_{\text {off }}$, the O- $V$ profiles after $-V_{\max }$ are distributed in the three zones of the device $(L, C$, and $R$ ) with no presence of sharp peaks. In this scenario, both interfaces $L$ and $R$ contribute to $M^{\mathrm{O}-V}$. Interestingly, after $V^{W}=V_{0}=0$ most $\mathrm{O}-V$ migrate to the $R$ interface, and the driving force for this movement is the depolarizing field pointing to the $R$ interface (we recall that $P$ points to the left interface $L$ after $\left.-V_{\max }\right)$. For $\tau_{W} / \tau_{\mathrm{off}}=1 / 99$ and $1 / 9$ this electromigration process is fast, reflected in a monotonous increase of $M^{\mathrm{O}-V}$ as the writing pulses go from $-V_{\max }$ to $V_{0}$. The final O- $V$ configuration suggests that the main contribution to $M^{\mathrm{O}-V}$ comes from the $L$ interface while the $R$ interface-with a high $\mathrm{O}-V$ density - presents a marginal contribution.

In the case of $\tau_{W} / \tau_{\text {off }}=1 / 4$ (longer writing pulses), the overall behavior is the same (transfer of $\mathrm{O}-V$ from $L$ to $R$ interfaces) but with a slower dynamics, which produces initially a slightly erratic curve followed by a nonmonotonic evolution of $M^{\mathrm{O}-V}$ when the writing voltage goes from $-V_{\max }$ to $-V_{0}$. Notice that between $-V_{\max }$ and $-V_{B}$, $M^{\mathrm{O}-V}$ decreases and further increases between $-V_{B}$ to $V_{0}$. This behavior reflects the competition between the $L$ and $R$ interfaces to the value of $M^{\mathrm{O}-V}$, the first (second) one increasing (decreasing) its contribution to the resistance as it becomes drained (filled) of O- $V$. Therefore, wider writing pulses delay the action of the depolarizing field, unveiling the described (more complex) $\mathrm{O}-\mathrm{V}$ dynamics.

Finally, Fig. 5(a) displays the evolution of the product $M^{\mathrm{O}-V} A^{F}$ as a function of $V^{W}$. In this case, both the ferroelectric and $\mathrm{O}-V$ electromigration (including the effect of the depolarizing field) memristive effects are included.

For $\tau_{W} / \tau_{\text {off }}=1 / 4$ (long writing pulses) the simulated $M^{\mathrm{O}-V} A^{F}$ displayed in Fig. 5(a) evidences that the remanent resistance values after $-V_{\max }$ and $-V_{0}$ are similar. In other words, the HSL exhibits a flat top part
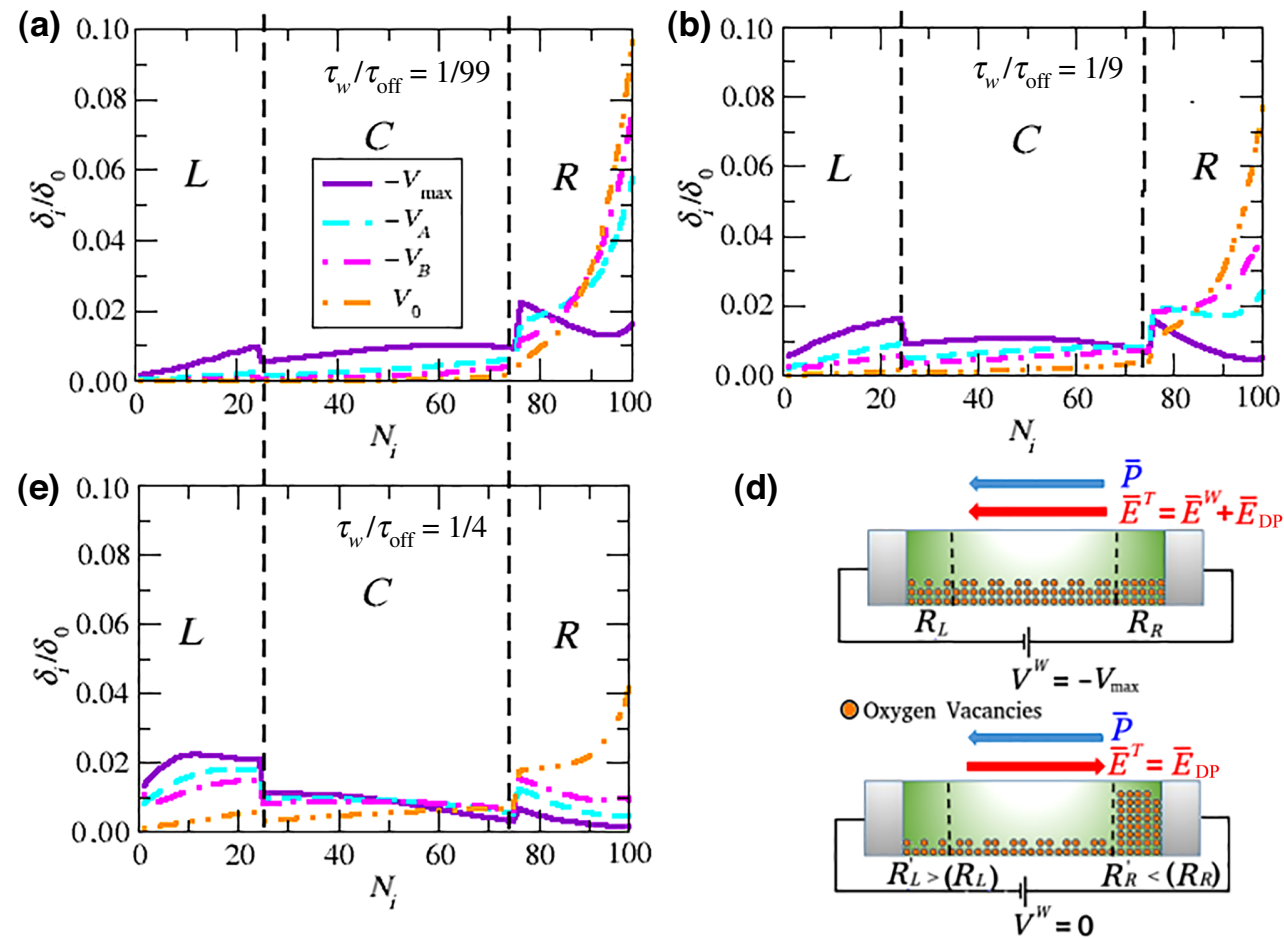

FIG. 4. O- $V$ profiles (rescaled by $\delta_{0}$ ) for $\tau_{W} / \tau_{\text {off }}=1 / 99$ (a), 1/9 (b), $1 / 4$ (c). The profiles with different colors follow the application of the writing voltages displayed in Fig. 3(b) (same color codes). (d) Sketch displaying the O- $V$ profile upon the application of $V^{W}=-V_{\max }$ (upper panel) and its evolution afterwards upon $V^{W}=V_{0}=0$ (lower panel). The electrical field $\bar{E}^{T}$ is the superposition of the external field $\bar{E}^{W}$ (pointing to the left in the upper panel and zero in the lower one) and the depolarizing field $\bar{E}_{\mathrm{DP}}$ (pointing to the right in both cases). O- $V$ migration from left $(L)$ to right $(R)$ is driven by $\bar{E}_{\mathrm{DP}}$. The device resistance increase is dominated by the decrease of $\mathrm{O}-V$ (increase of oxygen content) at the $L$ interface. 

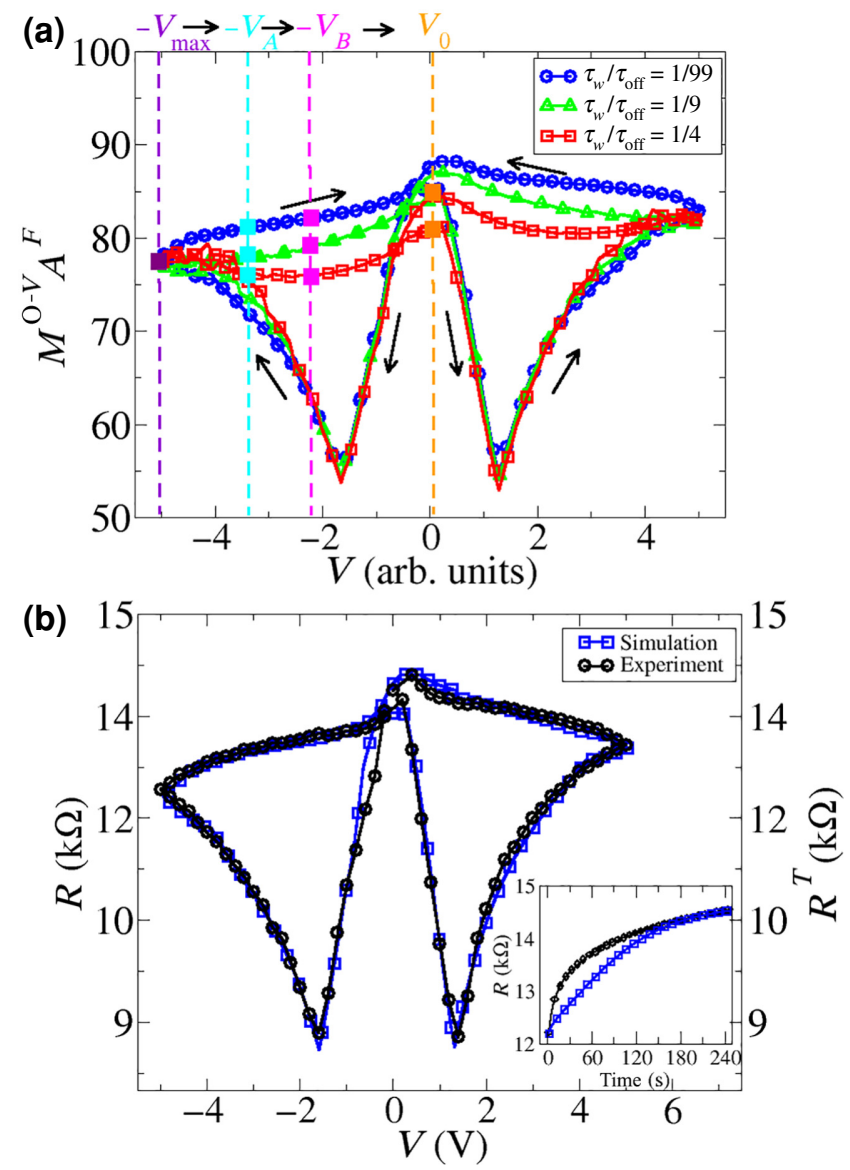

FIG. 5. (a) Evolution of $M^{\mathrm{O}-V} A^{F}$ vs $V^{W}$ for different $\tau_{W} / \tau_{\text {off }}$ ratios. (b) simulated HSL (right vertical axis) and experimental HSL (left vertical axis) corresponding to the $\mathrm{Pt} / \mathrm{PZT} / \mathrm{Pt}$ device [Fig. 1(e)]. In (a),(b) the simulations include the effect of O- $V$ electromigration due to both the external voltage and the depolarizing field together with the modulation of metal-ferroelectric barriers by the ferroelectric polarization. Inset: experimental (dark) and simulated (blue) relaxation of the resistance with time after a single write pulse $V^{W}$ followed by reading pulses. The numerical values of parameters are given in Table I. The conversion factor for $V^{W}$ between (a) and (b) is 1 arb. unit $=1 \mathrm{~V}$.

of the "table." For shorter writing pulses $-\tau_{W} / \tau_{\text {off }}=1 / 9$ and 1/99 - where the effect of the depolarizing field on $\mathrm{O}-V$ dynamics becomes largely dominant, we find that the remanent resistance values after $-V_{\max }$ is smaller than after $V_{0}$. This shows that the top part of the HSLs present nonzero slopes mimicking the experimental HSLs displayed in Figs. 1(e) and 1(f), respectively. This indicates that for our experimental systems, in the range of used writing stimulus (voltage and pulse time widths), O- $V$ dynamics is mainly controlled by the depolarizing field.

To test the consistency of our modeling, we notice that the maximum depolarizing field reached in the simulations is lower than the PZT device coercive field $\left(E_{\mathrm{DP}} \approx\right.$ $\left.3.9 \mathrm{MV} / \mathrm{m}<E_{C} \approx 6.3 \mathrm{MV} / \mathrm{m}\right)$, in order to describe a realistic situation where no strong sample depolarization takes place, as initially assumed. In addition, we recall that for perovskites with memristive mechanisms based solely on oxygen-vacancy electromigration, set and reset events are reported to be triggered by nominal electrical fields [defined as $V_{\text {set(reset) }} / d$, with $d$ being the separation between electrodes] ranging from approximately equal to $1 \mathrm{kV} / \mathrm{m}$ (ceramic manganite samples with contacts separated by a few $\mathrm{mm}$ ) [65] to approximately equal to $10 \mathrm{MV} / \mathrm{m}$ (outof-plane devices based on $100 \mathrm{~nm}$ manganite thin films) [66]. The $E_{\mathrm{DP}}$ we estimate for the modeling of the PZT system (approximately equal to $3.9 \mathrm{MV} / \mathrm{m}$ ) is close to the upper limit of the mentioned range. For the BTO device, the switching field approximately equal to $2 \mathrm{kV} / \mathrm{m}$ is also within the range of reported electrical fields able to drift diffuse $\mathrm{O}-V$. These considerations indicate that $\mathrm{O}-V$ electromigration is likely to occur in our experimental systems, as assumed.

With the evolution of $M^{\mathrm{O}-V} A^{F}$ vs $V^{W}$ already figured out, according to Eq. (1) we can fit the experimental HSL by determining the multiplicative constant $R^{\text {eff }}$, which depends on the initial $\mathrm{O}-V$ profile. We recall that as the experimental $R$ is an effective value dependant on the reading frequency $f$ (see Sec. II and the Supplemental Material [40]), $R^{\text {eff }}$ should also be rescaled properly for each experimental reading frequency. Figure 5(b) displays the $100 \mathrm{kHz}$ experimental HSL for the Pt/PZT/Pt system and the corresponding simulation. For the simulation we use $\tau_{W} / \tau_{\text {off }}=1 / 99$, which is very close to the experimental ratio $\left(\tau_{W} / \tau_{\text {off }}\right)_{\exp } \approx 0.012$ given by the used stimulus protocol (see Sec. II for details). For the voltage scale, we consider 1 arb. unit $=1 \mathrm{~V}$. The simulated curve displays an excellent agreement with the experimental data, with all the relevant features of the experimental HSL collected by the simulated one. We also notice that we constrain the quantitative simulation to the $\mathrm{Pt} / \mathrm{PZT} / \mathrm{Pt}$ system as the SRO/BTO/SRO device presents a slight asymmetric electrical response [see Fig. 1(f)], reflecting some asymmetry between both metal-oxide interfaces. A quantitative modeling of this system requires this issue to be reflected in the initial assumptions of the simulation and goes beyond the scope of the present paper.

Finally, we notice that in the scenario discussed above, where $\mathrm{O}-V$ drift is strongly driven by the depolarizing field acting in the absence of external stimuli, remanent resistance states should display time relaxations after the application of single write pulses. In order to explore this, we perform an additional experiment in our $\mathrm{Pt} / \mathrm{PZT} / \mathrm{Pt}$ system. A voltage writing pulse $[+6 \mathrm{~V}$, larger than the maximum voltage excursions of the HSL showed in Fig. 1(e)] is applied, followed by reading the effective remanent resistive states as a function of time (a small undisturbing dc pulse, with a superimposed ac signal of $100 \mathrm{kHz}$, is used to extract the remanent resistance values with the $L C R$ meter). 
The inset of Fig. 5(b) displays that, as expected, the remanent resistance relaxes with time, with a typical time scale of minutes, reflecting the action of the depolarizing field on O- $V$ electromigration. In the Supplemental Material [40] dc measurements performed in the same device are shown, displaying qualitatively similar relaxations to the ones observed with ac measurements. Also, the possible presence of capacitive artefacts accounting for the relaxations are discussed and discarded [40,67].

Upon the application of the $+6 \mathrm{~V}$ pulse the polarization points from right $(R)$ to left $(L)$, giving an O- $V$ profile, which is the mirror of the one displayed in Figs. 4(a) or 4(b). After the writing pulse is switched off, O- $V$ start to migrate from $R$ to $L$ and thus the device resistance starts to be dominated by and increases at the $R$ interface. The experimental relaxations are simulated using the model described above, as also shown in the inset of Fig. 5 . Very good agreement between the experimental and simulated curves for $t \geq 120 \mathrm{~s}$ after the pulse application is found. For shorter times, the experimental relaxation does not follow a pure exponential behavior - as is the case for the simulated curve - and this produces a slight divergence between both curves. This can be related to the existence of effects not included in our modeling, such as the presence of a charge trapping and detrapping process at the interfaces that could affect the device electrical transport and resistive memory behavior $[28,68,69]$. A quite similar resistance evolution (initial drop and further increase with time, not shown here) is found upon and after the application of negative single pulses, as expected given the device and the corresponding HSL symmetry.

\section{CONCLUSIONS}

In summary we find that, besides the modulation of the interfacial barriers by the direction of the ferroelectric polarization, the electromigration of $\mathrm{O}-V$ appears as a competing effect in the memristive response of symmetric metal-ferroelectric-metal devices. The driving force for O- $V$ electromigration is the depolarizing field linked to incomplete charge screening at ferroelectic-metal interfaces. With these ingredients, we are able to model and reproduce several nontrivial features of the experimental electrical behavior, including HSL and resistance relaxations. Our work sheds light on the nontrivial interplay between two relevant mechanisms that contribute to the resistance change in ferroelectric memristors, and in particular highlights the key role played by $\mathrm{O}-V$ electromigration. We recall that equivalent memristive responses are found in devices with different ferroelectric oxides (PZT and BTO) and geometries (out-of-plane and inplane configurations), suggesting that the physical scenario addressed in the paper is general for ferroelectric memristors with interfacial resistive switching. We recall that the combination of both nonvolatile and volatile (relaxations) resistance changes offers opportunities for the development of devices with neuromorphic capabilities, able to mimic the behavior of synapses and neurons, respectively, in a single device. Moreover, the volatile effect could be tailored by engineering the interfaces in order to control the screening lengths and tune the intensity of the depolarizing field.

\section{ACKNOWLEGMENTS}

We thank Dr. S. Matzen for the microfabrication of BTO devices and Dr. L. Steren for providing the PZT capacitor. We acknowledge support from INN-CNEA, UNCuyo (06/C591), ANPCyT (PICT2014-1382, PICT2016-0867, PICT2017-1836) and EU-H2020-RISE project "MELON" (SEP-2106565560). D.R. thanks the Nederlandse Organisatie voor Wetenschappelijk Onderzoek for a visiting grant, project 040.11.735, "Memcapacitive elements for cognitive devices."

[1] A. Sawa, Resistive switching in transition metal oxides, Mater. Today 11, 28 (2008).

[2] D. Ielmini and R. Waser, Resistive Switching: From Fundamentals of Nanoionic Redox Processes to Memristive Device Applications (Wiley-VCH, Weinheim, 2016).

[3] Zhongrui Wang, Saumil Joshi, Sergey E. Savel'ev, Hao Jiang, Rivu Midya, Peng Lin, Miao Hu, Ning Ge, John Paul Strachan, Zhiyong Li et al., Memristors with diffusive dynamics as synaptic emulators for neuromorphic computing, Nat. Mater. 16, 101 (2017).

[4] S. Yu, Neuro-Inspiring Computing Using Resistive Synaptic Devices (Springer International Publishing, Cham, 2017).

[5] André Chanthbouala, Vincent Garcia, Ryan O. Cherifi, Karim Bouzehouane, Stéphane Fusil, Xavier Moya, Stéphane Xavier, Hiroyuki Yamada, Cyrile Deranlot, Neil D. Mathur et al., A ferroelectric memristor, Nat. Mater. 11, 860 (2012).

[6] M. Ye Zhuravlev, Yong Wang, S. Maekawa, and Evgeny Y. Tsymbal, Tunneling electroresistance in ferroelectric tunnel junctions with a composite barrier, Appl. Phys. Lett. 95, 052902 (2009).

[7] E. Y. Tsymbal and H. Kohlstedt, Tunneling across a ferroelectric, Science 313, 181 (2006).

[8] Vincent Garcia, Stephane Fusil, Karim Bouzehouane, Shaima Enouz-Vedrenne, Neil D. Mathur, Agnes Barthelemy, and Manuel Bibes, Giant tunnel electroresistance for nondestructive readout of ferroelectric states, Nature 460, 81 (2009).

[9] V. Rouco, R. El Hage, A. Sander, J. Grandal, K. Seurre, X. Palermo, J. Briatico, S. Collin, J. Trastoy, K. Bouzehouane et al., Quasiparticle tunnel electroresistance in superconducting junctions, Nat. Commun. 11, 658 (2020).

[10] P. W. M. Blom, R. M. Wolf, J. F. M. Cillessen, and M. P. C. M. Krijn, Ferroelectric Schottky Diode, Phys. Rev. Lett. 73, 2107 (1994). 
[11] René Meyer and Rainer Waser, Hysteretic resistance concepts in ferroelectric thin films, J. Appl. Phys. 100, 051611 (2006).

[12] Lucian Pintilie, Viorica Stancu, L. Trupina, and Ioana Pintilie, Ferroelectric Schottky diode behavior from a $\mathrm{SrRuO}_{3}$ $\mathrm{Pb}\left(\mathrm{Zr}_{0.2} \mathrm{Ti}_{0.8}\right) \mathrm{O}_{3}-\mathrm{Ta}$ structure, Phys. Rev. B 82, 085319 (2010).

[13] J. E. Rault, G. Agnus, T. Maroutian, V. Pillard, Ph. Lecoeur, G. Niu, B. Vilquin, M. G. Silly, A. Bendounan, F. Sirotti et al., Interface electronic structure in a metal/ferroelectric heterostructure under applied bias, Phys. Rev. B 87, 155146 (2013).

[14] Andreas H. Hubmann, Shunyi Li, Sergey Zhukov, Heinz von Seggern, and Andreas Klein, Polarisation dependence of Schottky barrier heights at ferroelectric $\mathrm{BaTiO}_{3} / \mathrm{RuO}_{2}$ interfaces: Influence of substrate orientation and quality, J. Phys. D: Appl. Phys. 49, 295304 (2016).

[15] L. Pintilie, I. Vrejoiu, D. Hesse, G. LeRhun, and M. Alexe, Ferroelectric polarization-leakage current relation in high quality epitaxial $\mathrm{Pb}(\mathrm{Zr}, \mathrm{Ti}) \mathrm{O}_{3}$ films, Phys. Rev. B 75, 104103 (2007).

[16] S. Farokhipoor and B. Noheda, Screening effects in ferroelectric resistive switching of $\mathrm{BiFeO}_{3}$ thin films, APL Mater. 2, 056102 (2014).

[17] Xiaohui Liu, Yong Wang, John D. Burton, and Evgeny Y. Tsymbal, Polarization-controlled ohmic to Shottky transition at a metal/ferroelectric interface, Phys. Rev. B 88, 165139 (2013).

[18] L. Pintilie and M. Alexe, Metal-ferroelectric-metal heterostructures with Schottky contacts. I. Influence of the ferroelectric properties, J. Appl. Phys. 98, 124103 (2005).

[19] M. J. Rozenberg, M. J. Sánchez, R. Weht, C. Acha, F. Gomez-Marlasca, and P. Levy, Mechanism for bipolar resistive switching in transition-metal oxides, Phys. Rev. B 81, 115101 (2010).

[20] Kuibo Yin, Mi Li, Yiwei Liu, Congli He, Fei Zhuge, Bin Chen, Wei Lu, Xiaoqing Pan, and Run-Wei Li, Resistance switching in polycrystalline $\mathrm{BiFeO}_{3}$ thin films, Appl. Phys. Lett. 97, 042101 (2010).

[21] Diego Rubi, Fernando Gomez-Marlasca, Pierre Bonville, Dorothee Colson, and Pablo Levy, Resistive switching in ceramic multiferroic $\mathrm{Bi}_{0.9} \mathrm{Ca}_{0.1} \mathrm{FeO}_{3}$, Phys. B 407, 3144 (2012).

[22] Junjiang Tian, Zhengwei Tan, Zhen Fan, Dongfeng Zheng, Yadong Wang, Zoufei Chen, Fei Sun, Deyang Chen, Minghui Qin, Min Zeng et al., Depolarization-FieldInduced Retention Loss in Ferroelectric Diodes, Phys. Rev. Appl. 11, 024058 (2019).

[23] W. Lu, C. Li, L. Zheng, J. Xiao, W. Lin, Q. Li, X. R. Wang, Z. Huang, S. Zeng, K. Han et al., Multi-nonvolatile state resistive switching arising from ferroelectricity and oxygen vacancy migration, Adv. Mater 29, 1606165 (2017).

[24] H. J. Mao, C. Song, L. R. Xiao, S. Gao, B. Cui, J. J. Peng, F. $\mathrm{Li}$, and F. Pan, Unconventional resistive switching behavior in ferroelectric tunnel junctions, Phys. Chem. Chem. Phys. 17, 10146 (2015).

[25] Mengdi Qian, Ignasi Fina, Milena Cervo Sulzbach, Florencio Sánchez, and Josep Fontcuberta, Synergetic electronic and ionic contributions to electroresistance in ferroelectric capacitors, Adv. Electr. Mater. 5, 1800646 (2019).
[26] Milena Cervo Sulzbach, Saúl Estandía, Xiao Long, Jike Lyu, Nico Dix, Jaume Gàzquez, Matthew F. Chisholm, Florencio Sánchez, Ignasi Fina, and Josep Fontcuberta, Unraveling ferroelectric polarization and ionic contributions to electroresistance in epitaxial $\mathrm{Hf}_{0.5} \mathrm{Zr}_{0.5} \mathrm{O}_{2}$ tunnel junctions, Adv. Electr. Mater. 6, 1900852 (2019).

[27] Ming Li, Jian Zhou, Xiaosai Jing, Min Zeng, Sujuan Wu, Jinwei Gao, Zhang Zhang, Xingsen Gao, Xubing Lu, and J.-M. Liu, Controlling resistance switching polarities of epitaxial $\mathrm{BaTiO}_{3}$ films by mediation of ferroelectricity and oxygen vacancies, Adv. Electr. Mater. 1, 1500069 (2015).

[28] Aiping Chen, Wenrui Zhang, Liv R. Dedon, Di Chen, Fauzia Khatkhatay, Judith L. MacManus-Driscoll, Haiyan Wang, Dmitry Yarotski, Jun Chen, Xingsun Gao, Lane W. Martin, Andreas Roelofs, and Quanxi Jia, Couplings of polarization with interfacial deep trap and schottky interface controlled ferroelectric memristive switching, Adv. Funct. Mater. 2000664 (2020).

[29] Technical notes of Radiant AB capacitors, available at https://www.ferrodevices.com/1/297/files/TypicalPackaged ABPerformance.pdf.

[30] Arnoud S. Everhardt, Thibaud Denneulin, Anna Grünebohm, Yu-Tsun Shao, Petr Ondrejkovic, Silang Zhou, Neus Domingo, Gustau Catalan, Jiř́i Hlinka, Jian-Min Zuo et al., Temperature-independent giant dielectric response in transitional $\mathrm{BaTiO}_{3}$ thin films, App. Phys. Rev. 7, 011402 (2020).

[31] Arnoud S. Everhardt, Sylvia Matzen, Neus Domingo, Gustau Catalan, and Beatriz Noheda, Ferroelectric domain structures in low-strain $\mathrm{BaTiO}_{3}, \mathrm{Adv}$. Elect. Mater. 2, 1500214 (2016).

[32] Arnoud S. Everhardt, Silvia Damerio, Jacob A. Zorn, Silang Zhou, Neus Domingo, Gustau Catalan, Ekhard K. H. Salje, Long-Qing Chen, and Beatriz Noheda, PeriodicityDoubling Cascades: Direct Observation in Ferroelastic Materials, Phys. Rev. Lett. 123, 087603 (2019).

[33] Mei-Xiu Jia, Zhong-Qi Ren, Yan-Dong Liu, Yan Cheng, Rong Huang, Ping-Hua Xiang, Xiao-Dong Tang, BoBo Tian, Ni Zhong, and Chun-Gang Duan, Ferroelectric polarization-controlled resistive switching in $\mathrm{BaTiO}_{3} / \mathrm{SmNiO}_{3}$ epitaxial heterostructures, Appl. Phys. Lett. 114, 102901 (2019).

[34] J. F. Scott, Models for the frequency dependence of coercive field and the size dependence of remanent polarization in ferroelectric thin films, Integr. Ferroelectr. 12, 71 (1996).

[35] J. F. Scott, K. Watanabe, A. J. Hartmann, and R. N. Lamb, Device models for PZT/Pt, BST/Pt, SBT/Pt, and SBT/Bi ferroelectric memories, Ferroelectrics 225, 83 (1999).

[36] F. Chen, R. Schafranek, W. Wu, and A. Klein, Formation and modification of Schottky barriers at the PZT/Pt interface, J. Phys. D: Appl. Phys. 42, 215302 (2009).

[37] Xiaohui Liu, Yong Wang, J. D. Burton, and Evgeny Y. Tsymbal, Polarization-controlled ohmic to Schottky transition at a metal/ferroelectric interface, Phys. Rev. B 88, 165139 (2013).

[38] C. Ferreyra, M. J. Sánchez, M. Aguirre, C. Acha, S. Bengió, J. Lecourt, U. Lüders, and D. Rubi, Selective activation of memristive interfaces in $\mathrm{TaO}_{x}$-based devices by controlling oxygen vacancies dynamics at the nanoscale, Nanotechnology 31, 155204 (2020). 
[39] Wei Jin $\mathrm{Hu}$, Zhihong Wang, Weili Yu, and Tom Wu, Optically controlled electroresistance and electrically controlled photovoltage in ferroelectric tunnel junctions, Nat. Commun. 7, 1 (2016).

[40] See Supplemental Material at http://link.aps.org/supple mental/10.1103/PhysRevApplied.14.044045 for additional electrical characterization performed on the $\mathrm{Pt} / \mathrm{PZT} /$ Pt device.

[41] N. Novkovski and E. Atanassova, Frequency dependence of the effective series capacitance of metal $\mathrm{Ta}_{2} \mathrm{O}_{5} / \mathrm{SiO}_{2} \mathrm{Si}$ structures, Semicond. Sci. Technol. 22, 533 (2007).

[42] M. J. Rozenberg, I. H. Inoue, and M. J. Sánchez, Nonvolatile Memory with Multilevel Switching: A Basic Model, Phys. Rev. Lett. 92, 178302 (2004).

[43] D. Rubi, F. Tesler, I. Alposta, A. Kalstein, N. Ghenzi, F. Gomez-Marlasca, M. Rozenberg, and P. Levy, Two resistive switching regimes in thin film manganite memory devices on silicon, Appl. Phys. Lett. 103, 163506 (2013).

[44] Néstor Ghenzi, Marıa José Sánchez, and Pablo Levy, A compact model for binary oxides-based memristive interfaces, J. Phys. D: Appl. Phys. 46, 415101 (2013).

[45] D. J. Kim, J. Y. Jo, Y. S. Kim, Y. J. Chang, J. S. Lee, Jong-Gul Yoon, T. K. Song, and T. W. Noh, Polarization Relaxation Induced by a Depolarization Field in Ultrathin Ferroelectric batio 3 Capacitors, Phys. Rev. Lett. 95, 237602 (2005).

[46] J. Junquera and P. Ghosez, Critical thickness for ferroelectricity in perovskite ultrathin films, Nature 422, 506 (2003).

[47] F. Gunkel, D. V. Christensen, Y. Z. Chen, and N. Pryds, Oxygen vacancies: The (in)visible friend of oxide electronics, Appl. Phys. Lett. 116, 120505 (2020).

[48] S. B. Desu and D. P. Vijay, Novel fatigue-free layered structure ferroelectric thin films, Mater. Sci. Eng. B 32, 75 (1995).

[49] Y. Wang, Q. Y. Shao, and J.-M. Liu, Enhanced fatigueendurance of ferroelectric $\mathrm{Pb}_{1-x} \mathrm{Sr}_{x}\left(\mathrm{Zr}_{0.52} \mathrm{Ti}_{0.48}\right) \mathrm{O}_{3}$ thin films prepared by sol-gel method, Appl. Phys. Lett. 88, 122902 (2006).

[50] P. Hou, J. Wang, X. Zhong, and Y. Wu, A ferroelectric memristor based on the migration of oxygen vacancies, RSC Adv. 9, 13033 (2019).

[51] G. Y. Yang, E. C. Dickey, C. A. Randall, D. E. Barber, P. Pinceloup, M. A. Henderson, R. A. Hill, J. J. Beeson, and D. J. Skamser, Oxygen nonstoichiometry and dielectric evolution of $\mathrm{BaTiO}_{3}$. Part improvement of insulation resistance with reoxidation, J. Appl. Phys. 96, 7492 (2004).

[52] M. T. Chentir, E. Bouyssou, L. Ventura, G. Guégan, and C. Anceau, Electrical characterization and reliability of lanthanum dopec PZT thin film capacitors, Integr. Ferroelectr. 96, 75 (2008).

[53] S. M. Sze and Kwok K. Ng, Physics of Semiconductor Devices (John Wiley \& Sons, Ltd, New York, 2006).

[54] M. Stengel, P. Aguado-Puente, N. Spaldin, and J. Junquera, Band alignment at metal/ferroelectric interfaces: Insights and artifacts from first principles, Phys. Rev. B 83, 235112 (2011).

[55] X. Chen, N. J. Wu, J. Strozier, and A. Ignatiev, Direct resistance profile for an electrical pulse induced resistance change device, Appl. Phys. Lett. 87, 233506 (2005).

[56] H. Schraknepper, PhD Thesis, University of Aachen, 2015.

[57] S. B. Desu and D. P. Vijay, Novel fatigue-free layered structure ferroelectric thin films, Mater. Sci. Eng. B 32, 75 (1995).

[58] M. Dawber, P. Chandra, P. B. Litlewwod, and J. Scott, Depolarization corrections to the coercive field in thin-film ferroelectrics, J. Phys.: Condens. Matter 15, L393 (2003).

[59] S. V. Kalinin, Y. Kim, D. D. Fong, and A. N. Morozovska, Surface-screening mechanisms in ferroelectric thin films and their effect on polarization dynamics and domain structures, Rep. Prog. Phys. 81, 036502 (2018).

[60] Cristian Ferreyra, Wilson Román Acevedo, Ralph Gay, Diego Rubi, and María José Sánchez, Oxygen vacancy dynamics in redox-based interfaces: Tailoring the memristive response, J. Phys. D: Appl. Phys. 53, 015302 (2019).

[61] Tam T. Mayeshiba and Dane D. Morgan, Factors controlling oxygen migration barriers in perovskites, Solid State Ionics 296, 71 (2016).

[62] Carsten Funck, Christoph Bäumer, Stefan Wiefels, Tyler Hennen, Rainer Waser, Susanne Hoffmann-Eifert, Regina Dittmann, and Stephan Menzel, Comprehensive model for the electronic transport in $\mathrm{Pt} / \mathrm{SrTiO}_{3}$ analog memristive devices, Phys. Rev. B 102, 035307 (2020).

[63] Wei Jin Hu, Zhihong Wang, Weili Yu, and Tom Wu, Dielectric relaxation related to single-ionized oxygen vacancies in $\left(\mathrm{Pb}_{1-x} \mathrm{La}_{x}\right)\left(\mathrm{Zr}_{0.90} \mathrm{Ti}_{0.10}\right)_{1-x / 4} \mathrm{O}_{3}$ ceramics, Nat. Commun. 7, 1 (2016).

[64] R. R. Mehta, B. D. Silverman, and J. T. Jacobs, Depolarization fields in thin ferroelectric films, J. Appl. Phys. 44, 3379 (1973).

[65] N. Ghenzi, M. J. Sánchez, M. J. Rozenberg, P. Stoliar, F. G. Marlasca, D. Rubi, and P. Levy, Optimization of resistive switching performance of metal-manganite oxide interfaces by a multipulse protocol, J. Appl. Phys. 111, 084512 (2012).

[66] E. Miranda, W. Román Acevedo, D. Rubi, U. Lüders, P. Granell, J. Suñé, and P. Levy, Modeling of the multilevel conduction characteristics and fatigue profile of $\mathrm{Ag} / \mathrm{La}_{1 / 3} \mathrm{Ca}_{2 / 3} \mathrm{MnO}_{3} / \mathrm{Pt}$ structures using a compact memristive approach, J. Appl. Phys. 121, 205302 (2017).

[67] L. Pintilie, L. Hrib, I. Pasuk, C. Ghica, A. Iuga, and I. Pintilie, General equivalent circuit derived from capacitance and impedance measurements performed on epitaxial ferroelectric thin films, J. Appl. Phys. 116, 044108 (2014).

[68] L. Delimova, I. Grekhov, D. Mashovets, S. Shin, J. M. Koo, S. P. Kim, and Y. Park, A method of determining the charge trapped at the interfaces of a metal/ferroelectric/metal thinfilm structure, Phys. Solid State 48, 1182 (2006).

[69] D. S. Shang, Q. Wang, L. D. Chen, R. Dong, X. M. Li, and W. Q. Zhang, Effect of carrier trapping on the hysteretic current-voltage characteristics in $\mathrm{Ag} / \mathrm{La}_{0.7} \mathrm{Ca}_{0.3} \mathrm{MnO}_{3} / \mathrm{Pt}$ heterostructures, Phys. Rev. B 73, 245427 (2006). 